\title{
Structure and Mixing Properties of Combusting Monopropellant Sprays
}

\author{
T.-W. Lee* and G. M. Faeth $\dagger$ \\ University of Michigan, Ann Arbor, Michigan 48109
}

\begin{abstract}
The spray combustion properties of a hydroxylammonium nitrate (HAN)-based monopropellant (LGP 1845) were studied both theoretically and experimentally. Drop size, liquid mass flow rate, and liquid mass flux distributions were measured for pressure-atomized sprays in the atomization breakup regime, burning within a combustion gas environment at pressures of 4.5-5.0 MPa. Two separated-flow models were evaluated using the new measurements: a deterministic separated-flow model where drop-turbulence interactions were ignored, and a stochastic separated-flow model where drop-turbulence interactions were considered using random-walk computations for drop motion. When based on burning rates found from earlier single-drop experiments, both models were in reasonably good agreement with the measurements. Separated-flow effects are quite important for these sprays, with the length of the liquid-containing region being relatively independent of injector diameter and extending roughly $300 \mathrm{~mm}$ from the injector exit for injector exit velocities of roughly $70 \mathrm{~m} / \mathrm{s}$ and injector diameters of 0.3 and $0.6 \mathrm{~mm}$.
\end{abstract}

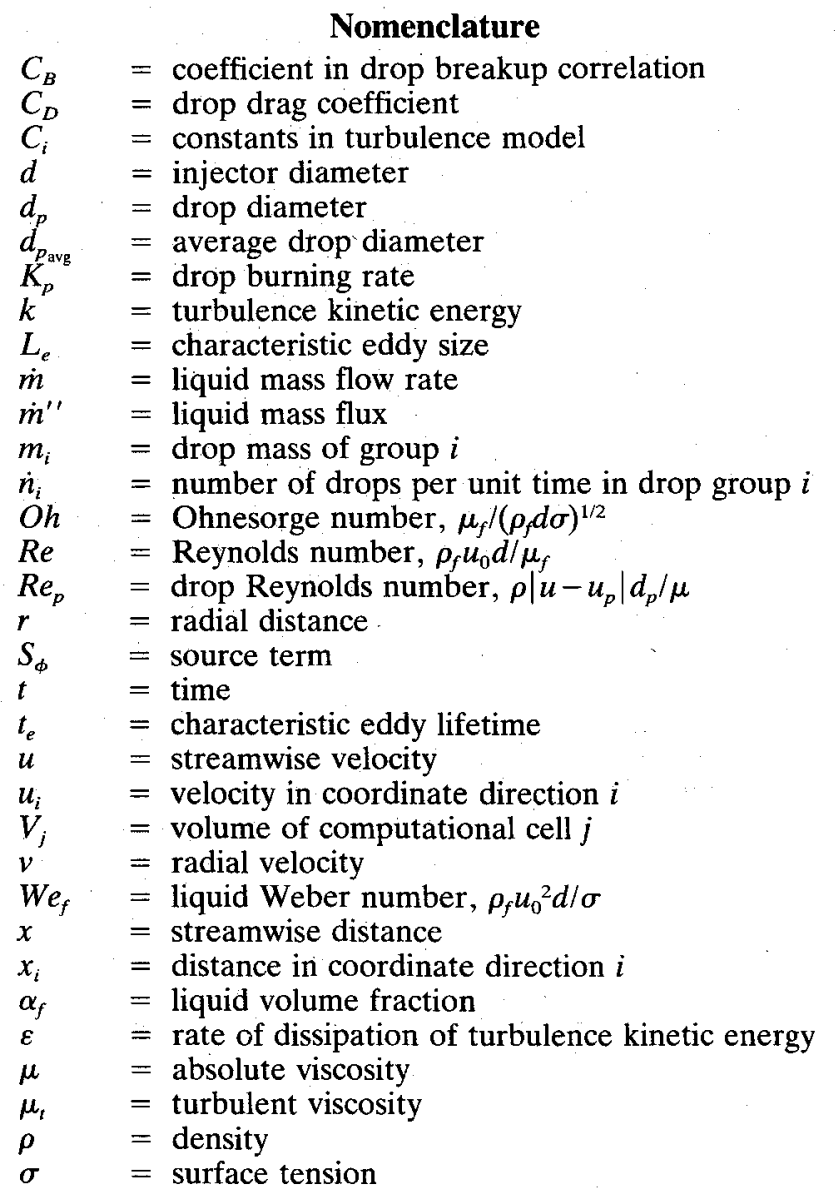

Received Dec. 30, 1989; presented as Paper 90-0463 at the AIAA 28th Aerospace Sciences Meeting, Reno, NV, Jan. 8-11, 1990; revision received Feb. 12, 1991; accepted for publication Feb. 21, 1991. Copyright (c) 1991 by the American Institute of Aeronautics and Astronautics, Inc. All rights reserved.

${ }^{*}$ Graduate Assistant; currently, Postdoctoral Fellow, Department of Mechanical Engineering, Pennsylvania State University, University Park, PA 16802.

$\nmid$ Professor, Department of Aerospace Engineering. Fellow AIAA.

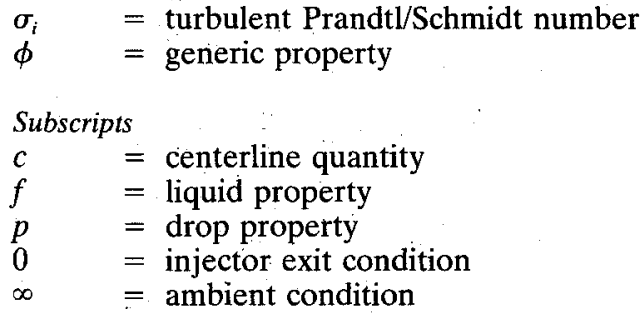

Superscripts

$\begin{aligned}\left({ }^{-}\right) & =\text {time-averaged quantity } \\ ()^{\prime}= & \text { time-averaged root-mean-squared fluctuating } \\ & \text { quantity } \\ \left(^{-}\right) \quad= & \text { number-averaged drop property }\end{aligned}$

\section{Introduction}

OMBUSTING monopropellant sprays represent an important fundamental multiphase combusting flow, because they are the premixed counterpart of more common spray diffusion flames. Additionally, combusting monopropellant sprays have applications to regenerative liquid-propellant guns, throttable thrustors, and underwater propulsion systems. Motivated by these considerations, the present investigation considered the structure of combusting pressureatomized monopropellant sprays both theoretically and experimentally, seeking to extend earlier work in this laboratory..$^{1-3}$ Similar to the earlier studies, ${ }^{1-3}$ the investigation was limited to a hydroxylammonium nitrate (HAN)-based monopropellant (LGP 1845) which is of interest for several highpressure monopropellant combustion systems.

The importance of multiphase flow phenomena is a significant issue for combusting monopropellant sprays. In particular, most applications involve high-pressure combustion (greater than $10 \mathrm{MPa}$ ) so that drops can approach their thermodynamic critical point where multiphase flow characteristics would disappear. Thus, combustor pressures required to reach near-critical conditions have been theoretically studied for HAN-based monopropellants. ${ }^{1}$ It was found that these pressures are unusually high, ca. $250 \mathrm{MPa}$ with an uncertainty of $50 \%$ due to uncertainties of thermochemical properties. As a result, multiphase effects are important for most applications involving these monopropellants.

The importance of finite interphase transport rates (separated-flow effects) is also a significant issue for high-pressure 
combusting sprays. This has been examined during 'earlier work in this laboratory. ${ }^{2,3}$ Initial work involved development of a locally homogeneous-flow (LHF) model for combusting HAN-based monopropellants, where interphase transport rates are assumed to be infinitely fast. ${ }^{2}$ This approach yielded reasonably good agreement with flow visualization measurements of Birk and Reeves ${ }^{4}$ for combusting LGP $1845 .{ }^{2}$ However, later evaluation in this laboratory suggested significant separated-flow effects using similar measurements as well as drop trajectory calculations where the gas velocity field was based on LHF predictions. ${ }^{3}$ Thus, the need for a separatedflow treatment of these sprays has been established although full separated-flow models of monopropellant sprays, allowing for effects of drop motion on the flow, have not been reported.

Information concerning the combustion properties of individual drops is needed to treat effects of separated flow; therefore, several studies relevant to drop combustion have been undertaken for the HAN-based monopropellants..$^{3,5-11}$ This includes measurements of strand burning rates, ${ }^{5-7}$ as well as observations of individual drop combustion in heated or combustion gas environments. ${ }^{3,8-11}$ Drop combustion at low pressures (less than $2 \mathrm{MPa}$ ) involves considerable subsurface liquid reaction, with bubble formation and mechanical removal of liquid by microexplosions being the main mechanism for the reduction of the drop diameter, ${ }^{3,8-11}$ This behavior follows because the liquid surface temperatures of HAN-based monopropellants are unusually high, ca. $1000 \mathrm{~K}$, tending to promote liquid-phase reactions. ${ }^{1}$ At higher pressures (greater than $2 \mathrm{MPa}$ ) within combustion gas environments, however, conventional surface gasification as a heterogeneous premixed flame is more dominant. ${ }^{3,5,6}$ The combined effect of the two mechanisms yields relatively high monopropellant drop burning rates, $10-40 \mathrm{~mm} / \mathrm{s}$, with a weak pressure dependence, ca. $p^{1 / 3}$, over the pressure range of $0.4-100 \mathrm{MPa} .^{3}$

Finally, recent studies of the near-injector (dense-spray) region of pressure-atomized sprays are helpful for identifying initial conditions for separated-flow predictions of spray properties. $^{12-16}$ It is generally agreed that this region involves a liquid core, somewhat like the potential core of single-phase jets, surrounded by a multiphase mixing layer. Flow properties approximate estimates based on the LHF approximation at high liquid volume fractions because the liquid dominates mixing properties due to its larger density; however, the bulk of the multiphase mixing layer exhibits significant effects of separated flow. ${ }^{12-16}$ Finally, when effects of liquid turbulence are small, average drop sizes near the liquid surface approximate estimates based on aerodynamic breakup theory due to Reitz and Bracco, ${ }^{12}$ while larger drops observed when liquid turbulence is present eventually break up to yield similar drop sizes. ${ }^{16}$

The present investigation sought to extend past work on the structure of combusting HAN-based monopropellant sprays. Spray combustion was observed in combustion gas environments near $5 \mathrm{MPa}$, using slide impaction to measure drop size, liquid mass flow rate, and liquid mass flux distributions. These measurements were used to evaluate separated-flow models of the process considering two limits, analogous to methods used for evaporating sprays ${ }^{17}$ : deterministic separated flow (DSF) where drop-turbulence interactions are ignored, and stochastic separated flow (SSF) were drop-turbulence interactions are considered using random-walk computations for drop motion. The present description of the study is brief; more details and tabulations of data can be found in Lee. ${ }^{18}$

\section{Experimental Methods}

\section{Apparatus}

The present spray combustion apparatus is similar to Lee et al. ${ }^{3}$ and Birk and Reeves. ${ }^{4}$ A sketch of the apparatus appears in Fig. 1. The experiments were conducted within a

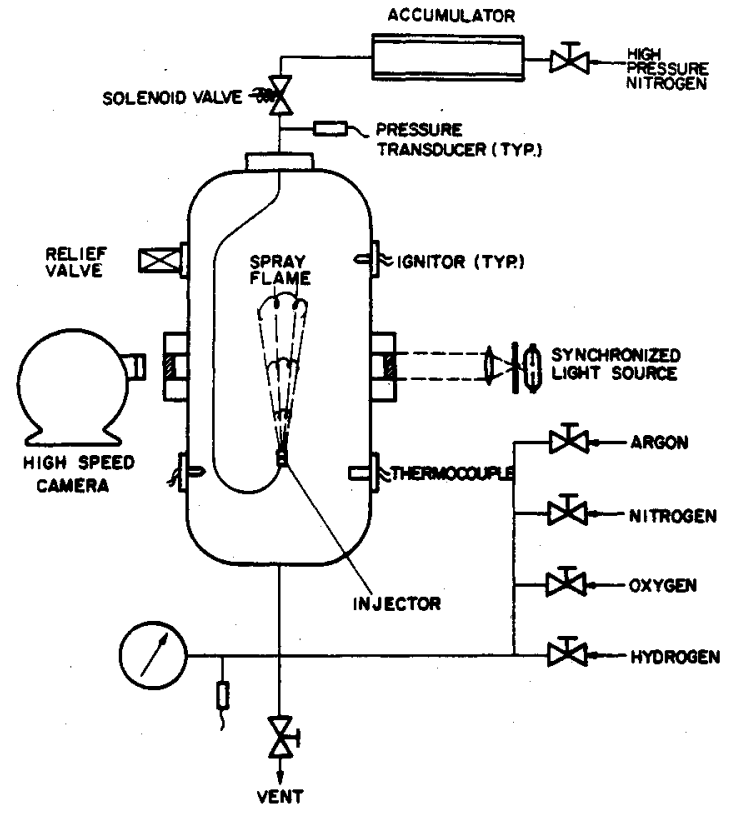

Fig. 1 Sketch of spray combustion apparatus.

windowed pressure vessel having an inside diameter and length of 130 and $430 \mathrm{~mm}$, respectively. The sprays were injected vertically upward in a combustion gas environment. The combustion gas environment was produced by filling the chamber with a combustible gas mixture and igniting it with a spark to achieve the combustion gas properties summarized in Table 1 (these properties were computed assuming thermodynamic equilibrium). ${ }^{19}$ The pressure of the combusting spray was set by adjusting the initial pressure of the combustible gas mixture because combustion of this gas approximated a constant volume process. ${ }^{3}$ The adiabatic combustion temperature of the gas mixture was somewhat greater than the adiabatic constantpressure combustion temperature of the monopropellant (see Table 1); however, temperature variations in this range do not have a large effect on drop burning rates. ${ }^{3}$ Additionally, these combustion gas temperatures are representative of conditions in liquid guns where combustion temperatures are increased due to constant volume combustion.

The spray was pressure-atomized using injectors having the diameters listed in Table 1 . The inlets of the injectors were both baffled to eliminate swirl, and smooth to.reduce effects of cavitation. Length-to-diameter ratios were 42 , yielding nearly fully developed turbulent pipe flow at the exit.

The apparatus operated by placing a 3-4-ml sample of the propellant in the injector feed line so that the passage was filled right up to the exit. A cap was placed over the exit to prevent gas inflow when the chamber was pressurized by filling or burning the combustion gas mixture. Injection was initiated by venting nitrogen from an accumulator into the delivery tube. Once the pressure of the propellant exceeded the chamber pressure, the cap popped off and the spray flowed into the combustion gas environment. The process ended when all the propellant was injected, after which the injector passage continued to be purged by nitrogen.

\section{Instrumentation}

The operation of the apparatus was monitored by measuring injector inlet and chamber pressures using pressure transducers. ${ }^{18}$ Earlier measurements involved flash shadowgraph motion pictures of the spray ${ }^{3}$; however, present measurements were limited to slide impaction.

A sketch of the slide impaction system appears in Fig. 2. The arrangement follows Ranz..$^{20}$ It involves a translating shield with a shutter slot, and a stationary probe on which a $\mathrm{MgO}$ layered glass slide $(12 \times 25 \mathrm{~mm})$ was mounted facing downward toward the injector. Initially, the translating shutter was 
Table 1 Summary of test conditions ${ }^{\mathrm{a}}$

\begin{tabular}{lccccccc}
\hline $\begin{array}{c}\text { Mea- } \\
\text { surements }\end{array}$ & $\begin{array}{c}\text { Injector } \\
\text { diameter, } \\
\text { mm }\end{array}$ & $\begin{array}{c}\text { Ambient } \\
\text { pressure, } \\
\text { MPa }\end{array}$ & $\begin{array}{c}\text { Pressure } \\
\text { drop, } \\
\text { MPa }\end{array}$ & $\begin{array}{c}\text { Injector } \\
\text { velocity, } \\
\text { m/s }\end{array}$ & $R e$ & $O h$ & $W e_{f}$ \\
\hline Drop size & 0.58 & 4.89 & 3.13 & 65.5 & 7800 & 0.030 & 54,200 \\
& 0.31 & 4.69 & 3.96 & 73.8 & 4700 & 0.041 & 36,700 \\
Liquid flux & 0.58 & 4.63 & 3.56 & 70.0 & 8300 & 0.030 & 61,700 \\
& 0.33 & 4.75 & 3.70 & 71.4 & 4800 & 0.040 & 36,500 \\
\hline
\end{tabular}

${ }^{a}$ Combustion of LGP 1845, consisting of $63.2 \%$ HAN, $20 \%$ TEAN, and $16.8 \% \mathrm{H}_{2} \mathrm{O}$ (by mass) Adiabatic combustion yields product temperature of $2150 \mathrm{~K}$, consisting of $69.2 \% \mathrm{H}_{2} \mathrm{O}, 12.9 \% \mathrm{CO}_{2}$ and $17.4 \% \mathrm{~N}_{2}$ (by volume). Liquid properties: $\rho_{f}=1454 \mathrm{~kg} / \mathrm{m}^{3}, \mu_{f}=0.0071 \mathrm{~kg} / \mathrm{ms}, \sigma=0.067 \mathrm{~N} / \mathrm{m}$. ${ }^{b}$ Injector length-to-diameter ratio of 42 , yielding fully developed turbulent pipe flow at exit.

Combustion product environment at $2790 \mathrm{~K}$ and consisting of $19.8 \% \mathrm{H}_{2} \mathrm{O}, 38.3 \% \mathrm{~N}_{2}$, and $40.9 \% \mathrm{Ar}$ (by volume).

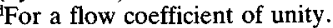

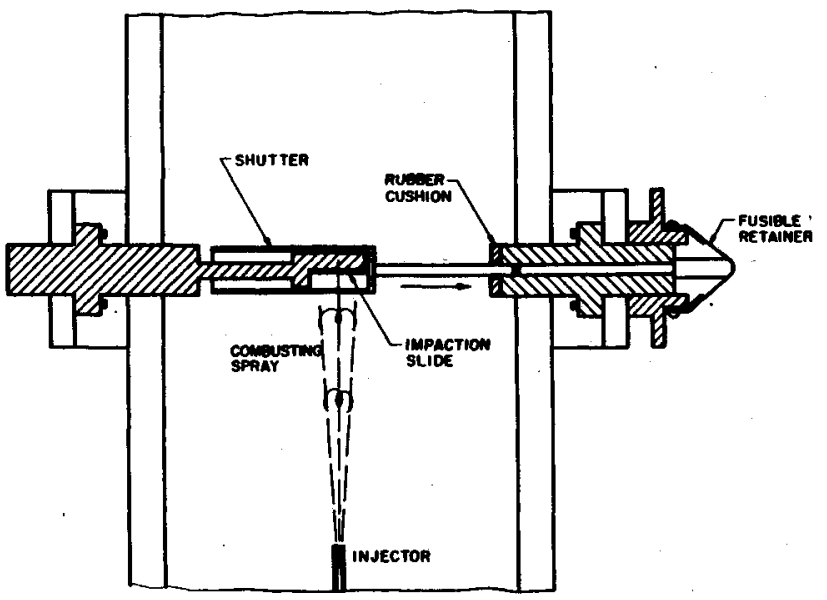

Fig. 2 Sketch of slide impactor assembly.

to the left of the slide (when viewed in Fig. 2) so that the slide was protected from initial disturbances by the shield. Once the spray was burned steadily, the wire retainer of the shield was fused by an electrical current, so that the unbalanced pressure force accelerated the shutter across the probe. The shutter opening ( $3.2 \mathrm{~mm}$ wide) traversing across the probe allowed drops to impact on the $\mathrm{MgO}$ layer, leaving cavities in the layer. The motion of the shutter was stopped by a rubber cushion after it had completely traversed the slide, leaving the slide protected once again by the shield. The motion of the shutter was recorded by a linear displacement transducer (not shown in Fig. 2) so that the time of passage across various portions of the slide was known.

The $\mathrm{MgO}$ layer was produced by passing the slide back and forth through the plume of a $\mathrm{Mg}$ flame from a $3 \times 25 \mathrm{~mm}$ $\mathrm{Mg}$ strip burning in air. Wetting the slide with water prior to coating it was found to produce a layer with reasonably good resistance to shock disturbances and the high-temperature environment. The relationship between drop and impact crater size is not affected significantly by drop velocity, ${ }^{20}$ and was found by calibrations using a Berglund-Liu monodisperse drop generator. The calibrations yielded a ratio of roughly 2.0 between the impact crater and drop diameters for drops up to $600 \mu \mathrm{m}$ in diameter. Crater diameters were measured using a Unitron inverted metallurgical microscope at $\times 50$ magnification, with a linear micrometer reticle scale on the eyepiece. Only drops having diameters larger than $10 \mu \mathrm{m}$ could be collected and sized due to limited collection efficiencies and resolution ${ }^{20}$; therefore, measured distributions are biased toward larger drop diameters, although this does not affect estimates of Sauter mean diameter (SMD) appreciably.

The slide impactor was positioned at various distances from the injector exit, by moving the injector. Due to excessively high liquid fluxes, which resulted in overlapping craters and washing out of the slide at the shortest feasible shutter-open- ing times, it was not possible to make measurements closer than $135 \mathrm{~mm}$ from the injector. By accumulating data for several slides at a particular condition, 50-200 drops were sized to find drop-size distributions and the SMD. Knowing the drop sizes and slide exposure times, these measurements could be used to find liquid fluxes and the total liquid flow rate over the cross section of the spray.

Experimental uncertainties of the slide impaction measurements were dominated by difficulties of overlapping impaction craters in the dense portion of the spray near the axis, and limitations of finite sample sizes. In particular, excessive liquid fluxes often required extrapolation of findings away from the axis to infer spray properties over the cross section. ${ }^{18}$ The experimental uncertainties (95\% confidence) were large as a result and are estimated as follows: SMD less than $25 \%$, liquid fluxes less than $40 \%$, and liquid flow rates only within a factor of two because flow rates near the axis involved extrapolation. These uncertainties are substantially greater than earlier work in evaporating air-atomized sprays at atmospheric pressure, ${ }^{17}$ reflecting the increased problems of the slide impaction technique in high-pressure and high-temperature environments within dense pressure-atomized sprays.

\section{Test Conditions}

Test conditions are summarized in Table 1 . Jet velocities in Table 1 are estimated using a flow coefficient of unity and are probably $15-20 \%$ too high based on calibrations of injectors having turbulent flow and similar length-to-diameter ratios. ${ }^{14-16}$ Injector Reynolds numbers are high enough to yield turbulent flow at the injector exit, well within the atomization breakup regime where the multiphase mixing layer beings to form right at the injector exit. ${ }^{2-16}$ Combustor pressures were in the range 4.6-4.9 $\mathrm{MPa}$, which is high enough for drop combustion to be dominated by surface gasification rather than microexplosions, based on single-drop combustion studies. $^{3}$

\section{Theoretical Methods}

\section{Gas-Phase Formulation}

In view of the large monopropellant burning rates for pressure test conditions, ca. $10 \mathrm{~mm} / \mathrm{s}$, the heterogeneous premixed flame that covers all liquid surfaces is relatively thin, ca. 1 $\mu \mathrm{m} .^{2}$ Thus the flow largely involves two states, unreacted liquid at its injection temperature, and completely reacted gaseous combustion products, if the ambient state also consists of products of adiabatic combustion. Since drop combustion properties were not strongly influenced by ambient temperatures, ${ }^{3}$ the ambient state was taken to be conditions after adiabatic combustion. Then, both phases have constant densities and because gas properties are uniform there is no need to treat scalar mixing in the gas phase; this substantially simplifies the formulation. The present approach involves Eulerian calculations for the properties of the gas phase and Lagrangian calculations of drop trajectories within the gas phase. Drop source times in the governing equations for the 
gas phase account for interphase transport of mass and momentum.

Major assumptions of the gas-phase formulation are as follows: axisymmetric and steady (in the mean) flow with no swirl, boundary-layer approximations, constant density gas, and negligible effects of buoyancy and liquid volume fraction variations. The first of these is a condition of the experiments while pressure-atomized sprays, like single-phase jets, generally satisfy the boundary-layer approximations. The constant-density approximation follows from the assumption of thin heterogeneous flames as discussed earlier. Additionally, injector velocities are ca. $70 \mathrm{~m} / \mathrm{s}$ while the multiphase flow region was only roughly $300 \mathrm{~mm}$ long; therefore, it is reasonable to neglect effects of buoyancy. Finally, recent findings for pressure-atomized nonevaporating sprays, ${ }^{14-16}$ show that liquid volume fractions are less than $1 \%$ outside of the liquid core, which is the region of interest for present separatedflow calculations. Similar to Solomon et al.,$^{17}$ the gas-phase velocity field was found using a $k-\varepsilon$ turbulence model that has been well calibrated for constant-density turbulent round jets. $^{21-23}$

Boundary conditions along the liquid core are specified as described later. Within the multiphase flow region, drop properties were found by solving Lagrangian equations of motion for the size and trajectories of a statistically significant sample of individual drops and then computing source terms for mass and momentum exchange from drops in the governing equations for the gas phase. This involves dividing the drops into $n$ groups (defined by initial position, size, and velocity) and then computing their subsequent motion in the flow. Effects of collisions do not appear to be important even in the dense portion of pressure-atomized sprays because liquid volume fractions are small outside the liquid core, as noted earlier; therefore, they are ignored. Similarly, effects of adjacent drops are not likely to influence the heterogeneous flames, because they are thin, and were ignored as well. Effects of secondary drop breakup may be important for present sprays ${ }^{16}$; however, this still was neglected due to the lack of information concerning this phenomenon. Because initial drop size distributions were based on measurements downstream of the region where secondary breakup would be important, the effect was accommodated in any event. Additionally, direct effects of drops on turbulence properties (called turbulence modulation) were ignored because the bulk of the flow is relatively dilute and methods to treat these phenomena are not well established..$^{23}$ Finally, drop-turbulence interactions are known to influence the structure of noncombusting sprays ${ }^{17,23}$; therefore, this was evaluated for combusting monopropellant sprays by carrying out both DSF and SSF computations, which ignore and consider drop-turbulence interactions as noted earlier.

Under present assumptions, the gas-phase governing equations are the same for the DSF and SSF formulations, and can be written as follows ${ }^{17.23}$ :

$$
r \partial / \partial x(\bar{\rho} \bar{u} \bar{\phi})+\partial / \partial r(r \bar{\rho} \bar{v} \bar{\phi})=\partial / \partial r\left(\left(r \mu_{t} / \sigma_{\phi}\right) \partial \bar{\phi} / \partial r\right)+r S_{\phi}
$$

where there is no need to consider Favre-averaged variables, because the density of the gas phase is constant and liquid volume fraction fluctuations are small. The parameters $\phi$ and $S_{\phi}$ appearing in Eq. (1) are summarized in Table 2, along with all empirical parameters used in the calculations. The Reynolds numbers of present flows are relatively high; therefore, laminar transport has been ignored. The source terms for $\phi=1$ (conservation of mass) and $\bar{u}$ in Table 2 are the drop source terms found by computing the net change of mass and momentum of each drop group $i$ passing through computational cell $j$, having a volume $V_{j}$. The boundary conditions of Eq. (1) involve a constant property ambient environment and symmetry at the axis (aside from the liquid core region which will be taken up later), as follows:

$$
r \rightarrow \infty, \quad \phi=0 ; \quad r=0, \quad \partial \phi / \partial r=0
$$

Table 2 Source terms and empirical constants for

\begin{tabular}{|c|c|c|c|c|c|c|}
\hline$\phi$ & \multicolumn{6}{|c|}{$S_{\phi}$} \\
\hline 1 & \multicolumn{6}{|c|}{$\sum_{i=1}^{n} \dot{n}_{i}\left(m_{i \mathrm{in}}-m_{i \text { out }}\right)_{j} / V_{j}$} \\
\hline $\bar{u}$ & \multicolumn{6}{|c|}{$\sum_{i=1}^{n} \dot{n}_{i}\left(\left(m_{i} u_{p i}\right)_{\mathrm{in}}-\left(m_{i} u_{p i}\right)_{\mathrm{out}}\right)_{j} / V_{i}$} \\
\hline$k$ & \multirow{2}{*}{\multicolumn{6}{|c|}{$\begin{array}{c}\mu_{t}(\partial \bar{u} / \partial r)^{2}-\bar{\rho} \varepsilon \\
\left(C_{\varepsilon 1} \mu_{t}(\partial \bar{u} / \partial r)^{2}-C_{\varepsilon 2} \bar{\rho} \varepsilon\right) \varepsilon / k\end{array}$}} \\
\hline$\varepsilon$ & & & & & & \\
\hline$\mu_{t}$ & $C_{\mu}$ & $C_{k 1}$ & $C_{\varepsilon 2}$ & $\sigma_{k}$ & $\sigma_{\varepsilon}$ & $K_{b}(\mathrm{~mm} / \mathrm{s})$ \\
\hline$C_{\mu} \bar{\rho} k^{2} / \varepsilon$ & 0.09 & 1.44 & 1.87 & 1.0 & 1.3 & 10 \\
\hline
\end{tabular}
separated-flow predictions

\section{Drop Formulation}

Drop trajectories were computed similar to earlier work. ${ }^{3}$ Drops were assumed to be surrounded by gas in the multiphase flow region. Effects of drop heatup were ignored and the drop radius was assumed to decrease at $10 \mathrm{~mm} / \mathrm{s}$, based on drop burning rate measurements at the pressure of present tests. ${ }^{3}$ In particular, the combustion process involves characteristic flame thicknesses on the order of $1 \mu \mathrm{m}$ in both the gas and liquid phases, ${ }^{2}$ so that bulk heating of drops is not relevant until they are too small (ca. $1 \mu \mathrm{m}$ diameter) to affect spray properties significantly. The high monopropellant burning rate implies a thin decomposition flame near the surface, well within the boundaries of the drop flow field; therefore, ambient gas properties were used to estimate drop drag and effects of forced convection on drop burning rates were ignored. Similarly, the eventual loss of the linear burning rate law for monopropellants $\left(K_{p}=-d r_{p} / d t=\right.$ constant $)$ for small drops was ignored, because this would occur for drop diameters of ca. $1 \mu \mathrm{m} .{ }^{3}$ Other assumptions were similar to earlier separated-flow models ${ }^{17,23}$ : the flow field around the drops was assumed to be quasisteady; virtual mass, pressure-gradient, Basset history and gravitational forces were ignored; drop swelling was ignored; and drop drag was estimated using the standard drag correlation for solid spheres.

Under these assumptions, the governing equations for drop motion are as follows ${ }^{23}$ :

$$
\begin{gathered}
d x_{p i} / d t=u_{p i}, \quad i=1,2,3 \\
d d_{p} / d t=-2 K_{p} \\
d u_{p i} / d t=\left(3 \rho \mathrm{C}_{D} / 4 d_{p} \rho_{p}\right)\left(u_{i}-u_{p i}\right)\left|u-u_{p}\right|, \quad i=1,2,3 \\
C_{D}=24\left(1+R e_{p}^{2 / 3} / 6\right) / R e_{p}, \quad R e_{p}<1000 ; \quad C_{D}=0.44 \\
R e_{p}>1000
\end{gathered}
$$

where a Cartesian coordinate system has been used and $\mid u$ $-u_{p} \mid$ denotes the magnitude of the relative velocity between the drop and the gas. The drag coefficient expression is identical to past work. ${ }^{23}$

Equations (3) and (5) were solved using time-averaged gasphase velocities for the DSF model. In contrast, the SSF model involves an approximate approach to treat turbulencedrop interactions that has been reasonably successful during past evaluations. ${ }^{17,23}$ This involves computing drop trajectories as they encounter a succession of turbulent eddies. The properties of each eddy are assumed to be uniform and to change randomly from one eddy to the next. The gas velocities within the eddies were formed by making a random selection from the probability density function (PDF) for velocity, assuming an anisotropic Gaussian PDF with streamwise and cross-stream variances of $k$ and $k / 2$. A drop was assumed to interact with an eddy as long as the time of interaction did 
not exceed the eddy lifetime $t_{e}$, or the relative displacement of the drop and the eddy did not exceed the characteristic eddy size $L_{e}$. These quantities were estimated, as follows ${ }^{23}$ :

$$
L_{e}=C_{\mu}^{3 / 4} k^{3 / 2} / \varepsilon, \quad t_{e}=L_{e} /(2 k / 3)^{1 / 2}
$$

All quantities needed for these procedures can be found from the gas-phase turbulence model.

\section{Initial Conditions}

Initial conditions were specified in the region of the liquid core as illustrated in Fig. 3. The present approach is somewhat similar to that of Chatwani and Bracco. ${ }^{13}$ Since the LHF approach is expected to give reasonable estimates to flow properties at large liquid volume fractions, ${ }^{14}$ the earlier LHF monopropellant combustion model was used to estimate the flowfield in the near-injector region. ${ }^{3}$ The boundary of the liquid core was then defined by locations where the timeaveraged liquid volume fraction was 0.997 , with mean and fluctuating streamwise and radial velocities identified at 40 locations from the LHF predictions (see Table 3 for representative data at these boundary points). The velocities of drop groups emanating from these positions were then estimated by either the mean velocities (DSF approach) or by a random selection from the velocity PDF's (SSF approach) of the LHF predictions. The drop-size distributions at the boundary of the liquid core were extrapolated back from the position nearest to the injector where they were measured, assuming an average drop velocity for the spray as a whole and the linear burning rate law (see Table 3 for the distribution used for $d=0.6 \mathrm{~mm}$ ). This translation procedure biases out drops that burn completely before reaching the first measurement location; however, measured spray properties are dominated by large drops and this effect is expected to be small. Finally, the mass flux through the time-averaged liquid volume fraction contour of 0.997 , for the LHF prediction, was used to prescribe drop mass fluxes at the boundary points.

Drop-size estimates obtained at the boundary of the liquid core in this manner appear to be reasonable. Using either aerodynamic breakup theory, according to Reitz and Bracco, ${ }^{12}$ or estimates based on maximum stable drop sizes for second-

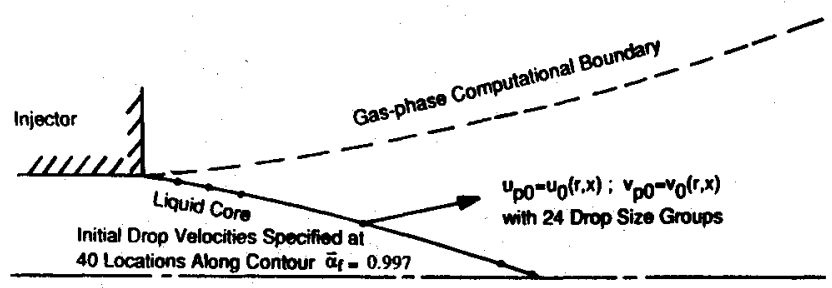

Fig. 3 Specification of initial conditions for separated-flow computations.

Table 3 Initial conditions for separated-flow predictions ${ }^{\text {a }}$

\begin{tabular}{cccccccc}
\hline \hline $\begin{array}{c}\text { Location } \\
\text { index }\end{array}$ & $x / d$ & $r / d$ & $\Delta \dot{m}_{i} / \dot{m}_{0}$ & $\bar{u}_{p} / u_{0}$ & $\bar{v}_{p} / u_{0}$ & $\bar{u}_{p}{ }^{\prime} / u_{0}$ & $\bar{v}_{p}{ }^{\prime} / u_{0}$ \\
\hline 1 & 0.31 & 0.47 & 0.0358 & 0.974 & 0.008 & 0.121 & 0.060 \\
10 & 3.13 & 0.39 & 0.0315 & 0.801 & 0.076 & 0.074 & 0.037 \\
20 & 6.25 & 0.29 & 0.0264 & 0.858 & 0.072 & 0.057 & 0.028 \\
30 & 9.37 & 0.15 & 0.0238 & 0.904 & 0.016 & 0.045 & 0.023 \\
40 & 10.8 & 0.00 & 0.0075 & 0.914 & 0.000 & 0.041 & 0.020 \\
\hline$d_{p 0}$ & $\%^{\mathrm{b}}$ & $d_{p 0}$ & $\%^{\mathrm{b}}$ & $d_{p 0}$ & $\%^{\mathrm{b}}$ & $d_{p 0}$ & $\%^{\mathrm{b}}$ \\
\hline 185 & 1.20 & 215 & 7.72 & 245 & 4.21 & 285 & 2.01 \\
190 & 2.91 & 220 & $\mathbf{6 . 9 2}$ & 250 & 3.91 & 295 & 1.71 \\
195 & 5.32 & 225 & 6.42 & 255 & 3.51 & 305 & 1.40 \\
200 & 7.92 & 230 & 5.72 & 260 & 3.21 & 315 & 1.10 \\
205 & 9.73 & 235 & 5.22 & 265 & 2.81 & 325 & 0.80 \\
210 & 8.73 & 240 & 4.71 & 275 & 2.31 & 335 & 0.50 \\
\hline
\end{tabular}

aDrop-size distribution for a 0.6-mm-diam injector.

${ }^{b}$ Relative number frequency (percent). ary breakup near the liquid surface, average drop sizes near the liquid surface for pressure-atomized nonevaporating sprays are correlated by ${ }^{16}$

$$
d_{p_{\text {avg }}}=C_{B} \sigma /\left(\rho u_{0}^{2}\right)
$$

where $C_{B}$ is roughly 10 and 40 for slug and fully developed flows at the injector exit. Taking the latter value as representative of present test conditions yields $d_{p_{\text {avg }}}=250 \mu \mathrm{m}$, which is comparable to present estimates of SMD along the boundary of the liquid core (see Table 3 ).

\section{Computations}

Calculations for the continuous phase were performed with a modified version of the GENMIX algorithm, ${ }^{24}$ with a computational grid similar to past work. ${ }^{17,23}$ Roughly 1000 drop groups were used for both the DSF and SSF predictions, with Eqs. (3-5) integrated by a second-order Runge-Kutte algorithm. Numerical accuracy was evaluated by doubling and halving the grid size and the number of drop groups, indicating that the results reported here are numerically closed within $3 \%$.

\section{Results and Discussion}

\section{Drop-Size Properties}

Predicted and measured SMD over the spray cross section are plotted as a function of $x / d$ in Fig. 4 for injector diameters of 0.3 and $0.6 \mathrm{~mm}$. Predictions include results from both the DSF and SSF models. The first observation is that liquid extends substantially beyond the region where liquid was seen during spray shadowgraph observations of liquid volume fractions, ${ }^{3}$ e.g., $x / d=500-1000$ for $d=0.3$ and $0.6 \mathrm{~mm}$, as opposed to $x / d$ ca. 350 for a similar range of pressures and injector diameters. It is clear that earlier spray shadowgraphs did not record drops within the dilute portions of the spray far from the injector exit and really do not offer a quantitative evaluation of predictions. Another feature of the measurements is that SMD does not scale with $x / d$ as $d$ is changed, which would be indicative of mixing-controlled behavior that might satisfy the LHF approximation. Instead, the scaling more closely approximates completion of combustion at a fixed distance from the injector with $x / d$ at the end of the liquid-containing region increasing from ca. 580 to ca. 980 as $d$ is reduced from 0.6 to $0.3 \mathrm{~mm}$. Because initial drop sizes and velocities of the two injectors are estimated to be nearly the same, this behavior is indicative of strong effects of separated flow.

Predictions using the DSF and SSF models are not very different from each other in Fig. 4 and are in reasonably good agreement with the measurements. The agreement between predictions and measurements is best toward the downstream end of the spray where the slide impaction technique was more reliable due to lower liquid fluxes. Discrepancies between predictions and measurements are largest for nearinjector positions and $d=0.3 \mathrm{~mm}$, where drop sizes are felt to be biased downward because of difficulties of measuring large drops near the axis due to high rates of drop impaction since these positions were relatively close to the injector exit. The predictions were strongly dominated by effects of drop burning rates, while initial drop-size distributions were calibrated; therefore, present findings suggest that drop burning rates observed in the combusting sprays are consistent with the single-drop results reported earlier. ${ }^{3}$ As discussed later, however, reducing the drop burning rate by roughly one-third from the value measured in Ref. 3 would yield better agreement between present predictions and measurements. Nevertheless, a change of this magnitude is not large in view of the approximations of the predictions and the uncertainties of the measurements.

Measured and predicted (both DSF and SSF methods) dropsize distributions are illustrated in Figs. 4 and 5 for the two 


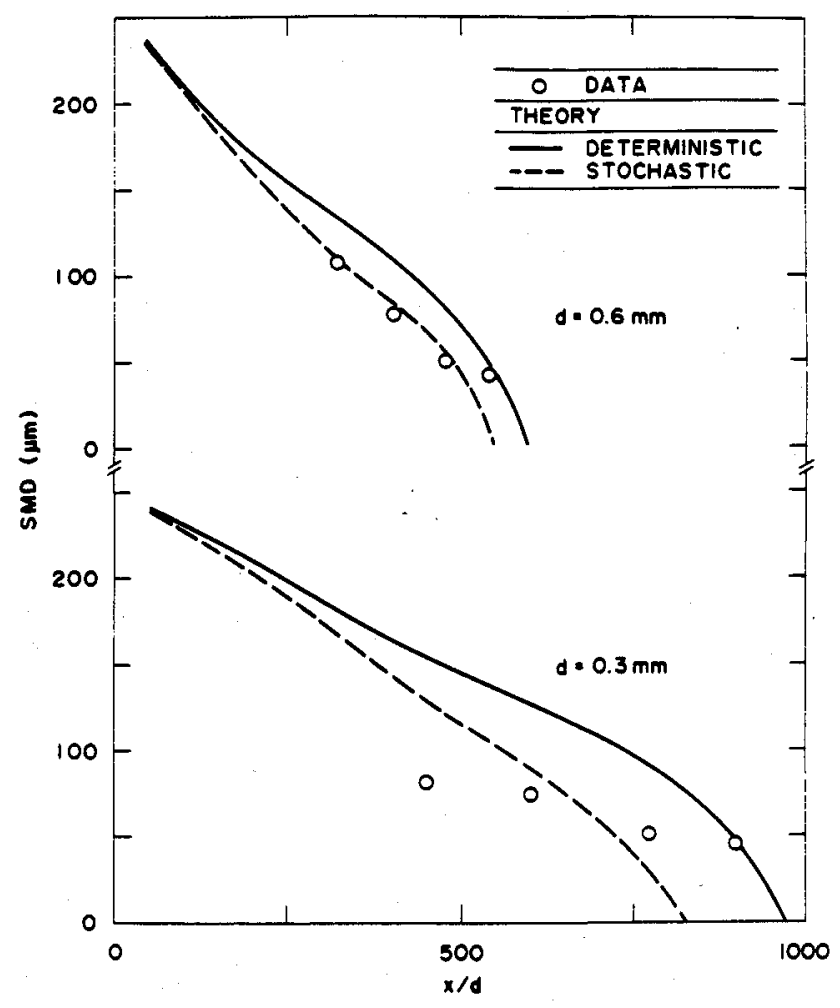

Fig. 4 Predicted and measured SMD variation with streamwise distance.

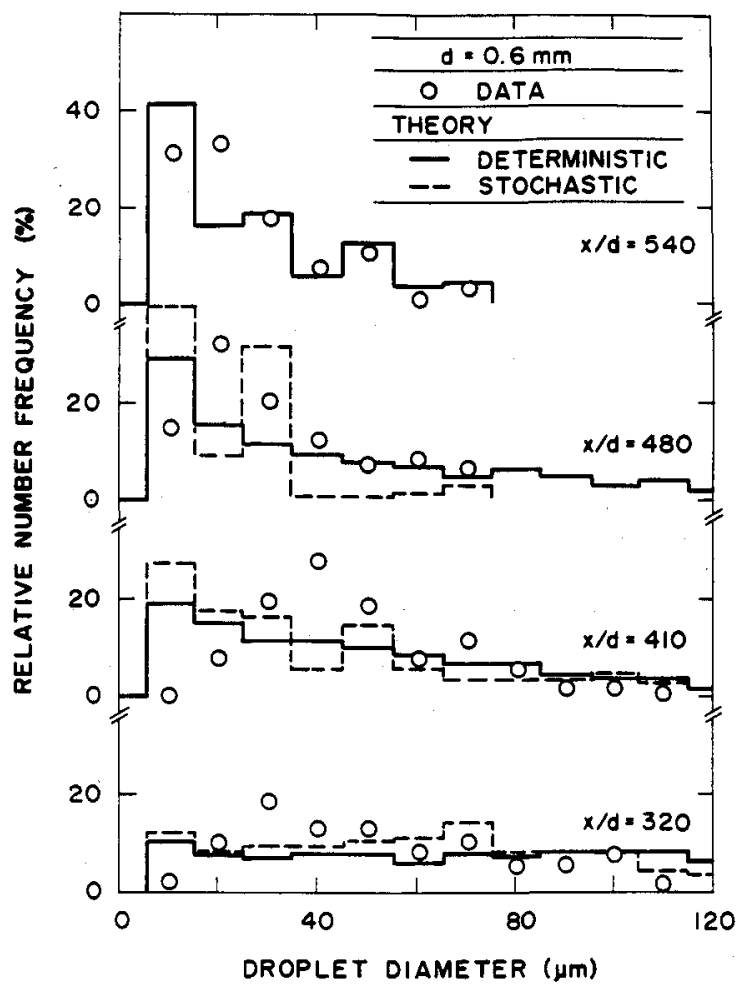

Fig. 5 Predicted and measured drop-size distributions at various streamwise distances, $d=0.6 \mathrm{~mm}$.

injector diameters. As noted earlier, the measurements at positions nearest the injector were extrapolated to the injector exit in an approximate manner to provide initial conditions for the predictions. Reversing the process to yield the predictions illustrated in Figs. 5 and 6 does not exactly reproduce the distributions at the smallest $x / d$ due to different rates of deceleration experienced by various drop groups.

Aside from limitations in resolving drop sizes near $10 \mu \mathrm{m}$, the subsequent evolution of the drop-size distributions with

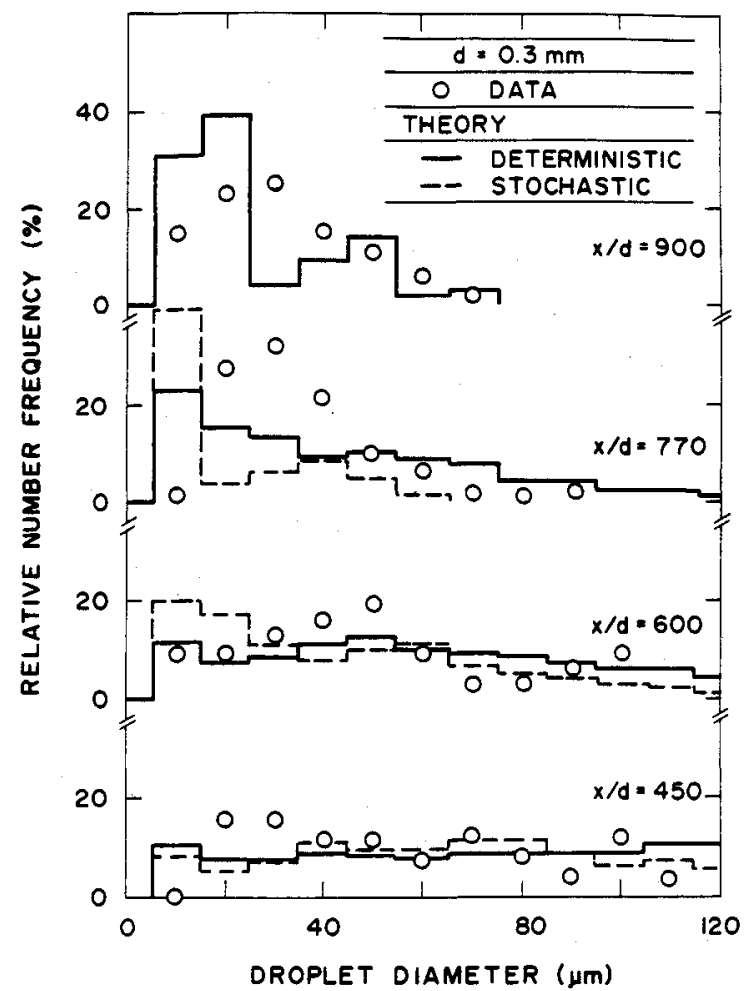

Fig. 6 Predicted and measured drop-size distributions at various streamwise distances, $d=0.3 \mathrm{~mm}$.

increasing $x / d$ is also predicted reasonably well in Figs. 5 and 6. In particular, the distributions become skewed toward smaller drop sizes as the end of the liquid-containing region is approached, which is represented reasonably well by the predictions.

\section{Liquid Flow Properties}

As noted earlier, measurements of liquid flow rates and fluxes are less accurate than measurements of SMD; however, in spite of these limitations, the results help provide a more complete picture of the structure of the sprays. Measured and predicted normalized liquid flow rates over the spray cross section are plotted as a function of $x / d$ in Fig. 7 for the two injectors. Measurements at one position for each of the injectors are significantly higher than the rest and are felt to be anomalous due to problems with the slide impactor in the period where these results were obtained. The remaining results show the expected trend of decreasing liquid flow rates with increasing $x / d$. Although liquid is observed for $x / d>$ 500 , the flow rate is generally less than $10 \%$ of the original flow rate at the injector exit. This comes about due to both consumption of drop liquid, as well as reduced drop velocities in the relatively slow gas flow far from the injector.

The comparison between predictions and measurements for liquid flow rates in Fig. 7 is not as good as for SMD in Fig. 4. This is typical of evaluations of spray models using liquid flow rates and fluxes: these quantities are difficult to measure accurately in pressure atomized sprays and are particularly sensitive to uncertainties in model predictions. ${ }^{15,17,23}$ Nevertheless, these findings suggest that the drop burning rate constant used during present computations may be too large. In particular, a value roughly one-third smaller would yield reasonably good agreement between predictions and measurements of liquid flow rates in Fig. 7, and improved SMD predictions in Fig. 4, as noted earlier. As before, there is little to choose between the DSF and SSF methods, with the latter yielding a somewhat shorter spray length. This comes about due to higher mean drag when instantaneous gas velocities are used to estimate drop trajectories (because drag forces are nearly quadratic functions of relative velocities for present 


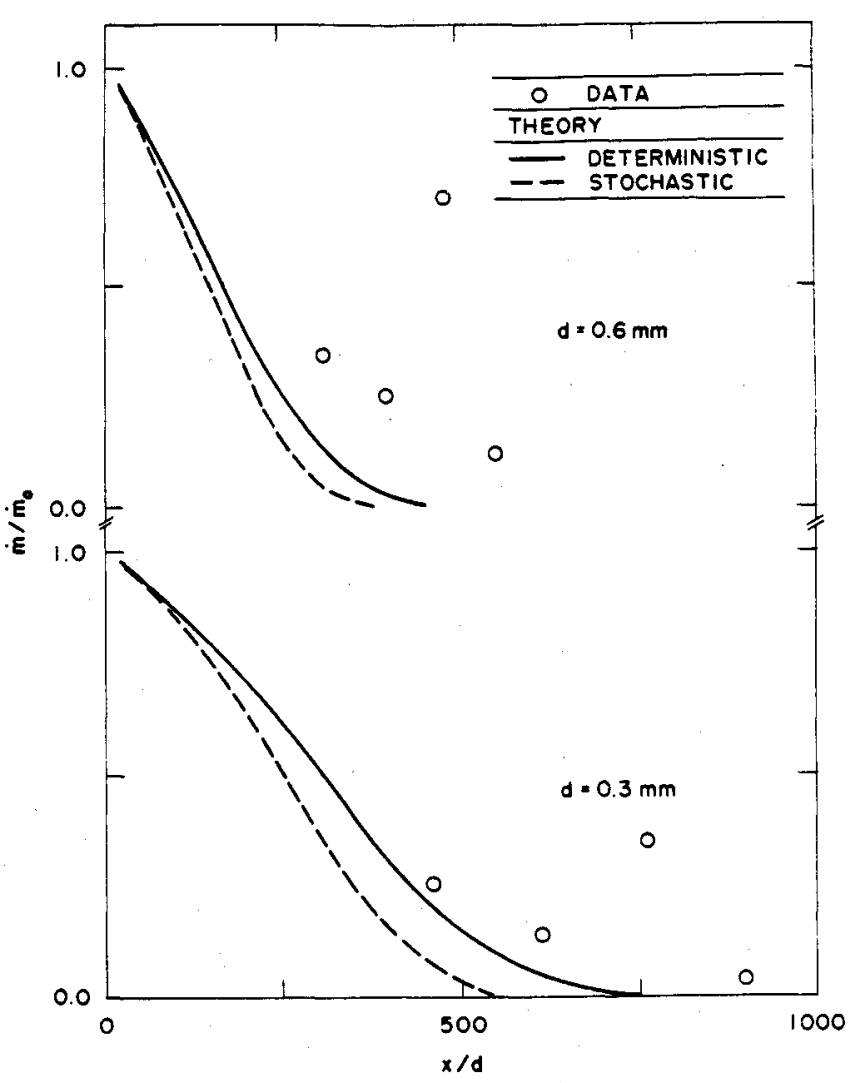

Fig. 7 Predicted and measured liquid mass flow rate variation with streamwise distance.

conditions), as well as effects of turbulent dispersion which transports drops laterally into slower moving gas near the edge of the flow. However, effects of turbulence-drop interactions are much reduced for the present monopropellant sprays in comparison to evaporating sprays, ${ }^{17}$ because the rate of drop gasification is not influenced by the radial position of the drops within the flow.

An indication of the width of the sprays can be seen from the plot of normalized liquid mass flux in Fig. 8 for $d=0.3$ $\mathrm{mm}$. Results for $d=0.6 \mathrm{~mm}$ are similar. ${ }^{18}$ These results are plotted in terms of the radial similarity variable for turbulent jets, $r / x$, so that the actual width of the flow can be seen. However, similarity of liquid mass fluxes in these coordinates is neither expected nor observed. Spray widths extend to $r / x$ ca. 0.12 at $x / d=460$, but the liquid becomes progressively more confined near the axis at larger distances from the injector.

Liquid fluxes are difficult to predict accurately, as noted earlier, ${ }^{15,17,23}$ and there is only qualitative agreement between predictions and measurements in Fig. 8. In fact, predictions underestimate the spray length for these conditions (see Figs. 4 and 7), so that no predictions are available for comparison with the measurements at the highest position. As before, use of a lower drop burning rate constant would help resolve these difficulties. Consideration of turbulent dispersion using the SSF model does not yield a wider liquid flux distribution. Instead, separated-flow effects dominate the process, causing small drops to disappear more quickly in the low-velocity region near the edge of the flow.

\section{Additional Predictions}

The comparison between predictions and measurements indicates that both the DSF and SSF models provide the general features of the combusting monopropellant sprays, particularly the drop-size properties where predictions are less sensitive to model parameters and measurements are more reliable. Thus, additional computations were preformed to help gain insight concerning spray properties where meas-

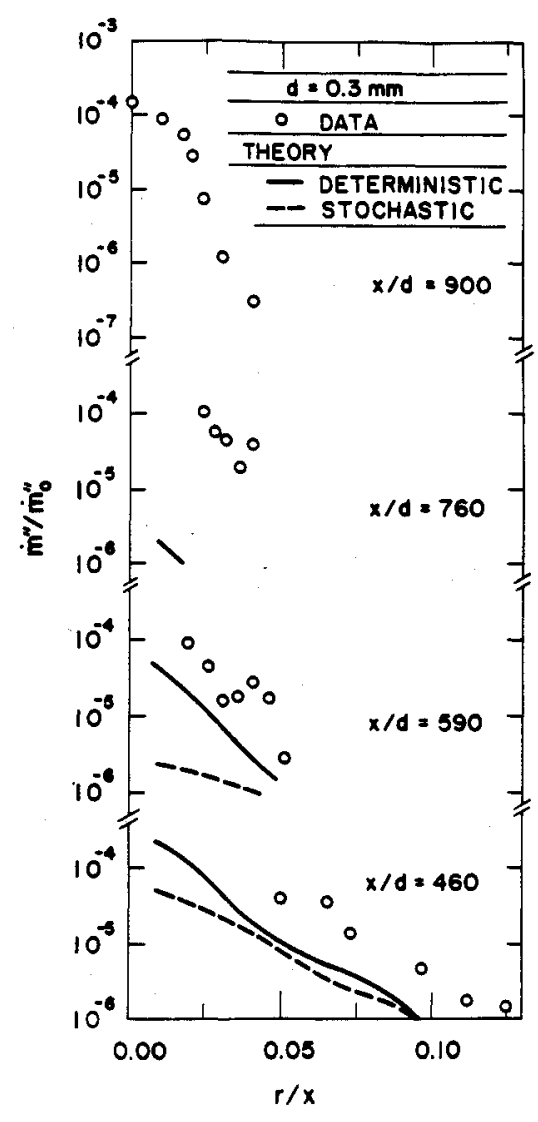

Fig. 8 Predicted and measured liquid mass flux distributions at various streamwise distances, $d=0.3 \mathrm{~mm}$.

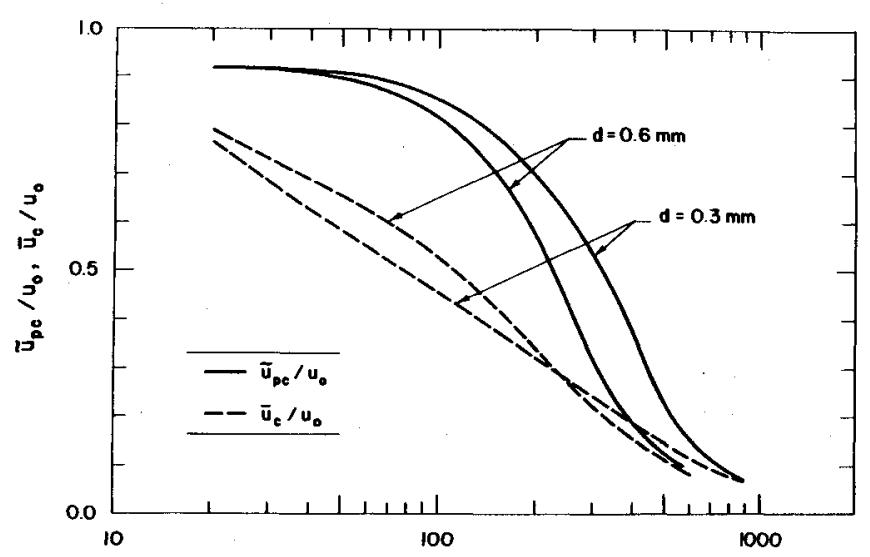

Fig. 9 Predicted mean phase-velocity variations with streamwise distance along the axis.

urements were not available. This included predictions of phase velocities and the sensitivity of predictions to variations of model parameters and spray operating conditions.

Predicted phase velocities for present test conditions are plotted in Fig. 9. Number-averaged drop velocities and timeaveraged gas velocities along the axis, found from the DSF model, are plotted as a function of distance from the injector for $d=0.3$ and $0.6 \mathrm{~mm}$. Effects of separated flow are evident, with liquid velocities decaying much more slowly than gas velocities. The penetration length of the spray, in terms of $x /$ $d$, and relative velocities are larger for $d=0.3 \mathrm{~mm}$, because the smaller diameter injector involves higher rates of deceleration of the gas, which cannot be followed by the drops due to their limited response properties. In fact, liquid velocities remain nearly constant until $x / d>100$, because both gas velocities and drop diameters are largest in this region. Farther from the injector, drop velocities decrease rapidly toward 
Table 4 Parameter sensitivity of separated-flow predictions ${ }^{\mathrm{a}}$

\begin{tabular}{lccc}
\hline \hline & $\begin{array}{c}\text { Factor of } \\
\text { variation }\end{array}$ & \multicolumn{2}{c}{ Percent increase of $x / d$} \\
\cline { 2 - 4 } Input parameter & $1 / 2$ & 41 & $\dot{\mathrm{SMD}=0}$ \\
\hline Drop burning rate & 2 & -32 & -40 \\
Drop burning rate & $1 / 2$ & -28 & -38 \\
Initial SMD & 10 & -42 & -30 \\
Ambient density & 10 & -80 & -84 \\
Injector diameter & - & -9 & -11 \\
Turbulent & & & \\
\multicolumn{1}{c}{ dispersion } & & & \\
\hline \hline
\end{tabular}

Results for $d=0.6 \mathrm{~mm}$ using the DSF model except as noted.

${ }^{b}$ The effect of including turbulent dispersion is shown.

gas velocities, due to increase 4 drop response as the drops become smaller. This increases drop residence times for a particular penetration length and makes estimates of properties like liquid flow rates and fluxes at large $x / d$ very sensitive to model parameters, as noted earlier.

The sensitivity of predictions to variations of model parameters and spray operating conditions can be seen more quantitatively from the results summarized in Table 4 . The percent increase of $x / d$ at the points where SMD $=0$ and $\dot{m} / \dot{m}_{0}=$ $\frac{1}{2}$ are tabulated for various changes of parameters. These results involve use of the DSF model (except for the last item) and $d=6.0 \mathrm{~mm}$; however, they are typical.

As expected, results in Table 4 show that the drop burning rate has a strong effect on the axial penetration of spray liquid, with a nearly one-to-one correspondence for changes of drop burning rates by a factor of two. Although not shown, varying the initial velocity behaves in a manner reciprocal to the burning rate constant because the product of the residence time (which is inversely proportional to velocity) and the burning rate yields the variation of drop diameter for monopropellants. Results are less sensitive to initial SMD, however, because reduction of drop sizes increases momentum exchange to the gas causing gas velocities to increase. The resulting larger drop velocities tend to compensate for smaller drop lifetimes when evaluating spray penetration. Raising the ambient density by a factor of 10 , for a fixed initial SMD, only decreases penetration lengths by $30-40 \%$, largely due to increased entrainment rates which reduce gas-phase velocities in the spray. Varying ambient density is actually likely to have a greater effect, however, by reducing drop sizes through the drop breakup criterion, e.g., Eq. (8). Based on effects of SMD in Table 4, this would considerably shorten spray penetration lengths from results measured during the present investigation. Effects of injector diameter variations follow from the nearly constant spray penetration length for various injector diameters, i.e., increasing the diameter by a factor of 10 reduces the penetration length, in terms of $x / d$, by nearly the same ratio. Finally, effects of including turbulent dispersion are relatively small, particularly in view of uncertainties in estimating initial conditions for separated-flow calculations.

\section{Conclusions}

Major conclusions of the study are as follows:

1) Present slide impaction measurements show that the liquid-containing region is two to four times larger than found during earlier measurements using flash shadowgraphs by Lee et al. ${ }^{3}$ due to the inability of the shadowgraph approach to detect dilute portions of the spray.

2) Present measurements of combusting spray properties show that monopropellant drop burning rates within the sprays, $-d r_{p} / d t$ ca. $10 \mathrm{~mm} / \mathrm{s}$, are consistent with earlier single drop burning rate measurements at the same pressure, ca. $5 \mathrm{MPa}$, although a value roughly one-third smaller would have yielded better quantitative agreement between present measurements and predictions for sprays. Due to its dominance of spray combustion properties, additional study of the drop combus- tion properties of HAN-based monopropellants is recommended.

3) The combusting propellant sprays exhibited strong effects of separated flow, with the length of the liquid-containing region being nearly the same, ca. $300 \mathrm{~mm}$, for injector diameters of 0.3 and $0.6 \mathrm{~mm}$ when initial SMD and injector exit velocities were nearly the same.

4) Estimated mean drop sizes near the injector exit were consistent with recent measurements for nonevaporating pressure-atomized sprays. ${ }^{16}$ This suggests that initial mean drop sizes (SMD) can be estimated from either the aerodynamic breakup theory of Reitz and Bracco, ${ }^{12}$ or analogous secondary breakup criteria, ${ }^{16}$ through Eq. (8). However, this approach is only provisional, pending additional study of effects of liquid turbulence at the injector exit and approach of the flow to the LHF limit.

5) Predictions using the DSF and SSF models were in reasonably good agreement with each other and with the measurements, particularly in view of the relatively large uncertainties of estimates of initial drop properties and the slide impaction measurements. Thus, the DSF approach is favored, because it requires less extensive computations. The relatively small effects of drop-turbulence interactions follows since the gas phase has constant properties and drop burning rates are relatively independent of the properties of their surroundings for combusting monopropellant sprays.

\section{Acknowledgments}

This research was sponsored by the Army Research Office and the U.S. Army Armament Research, Development and Engineering Center, Picatinney Arsenal, NJ under Contract DAAL03-86-K-0154.

\section{References}

${ }^{1}$ Kounalakis, M. E., and Faeth, G. M., "Combustion of HANBased Liquid Monopropellants near the Thermodynamic Critical Point," Combustion and Flame, Vol. 74, Nov. 1988, pp. 179-192.

${ }^{2}$ Lee, T.-W., Gore, J. P., Faeth, G. M., and Birk, A. "Analysis of Combusting High-Pressure Monopropellant Sprays," Combustion Science and Technology, Vol. 57, 1988, pp. 95-112.

${ }^{3}$ Lee, T.-W., Tseng, L.-K., and Faeth, G. M., "Separated-Flow Considerations for Pressure-Atomized Combusting Monopropellant Sprays," Journal of Propulsion and Power, Vol. 6, No. 4, 1990, pp. 382-391.

${ }^{4}$ Birk, A., and Reeves, P., "Annular Liquid Propellant Jets-Injection, Atomization and Ignition," Rept. BRL-TR-2780, Ballistic Research Laboratory, Aberdeen Proving Ground, March 1987.

${ }^{5}$ McBratney, W. V., "Windowed Chamber Investigation of the Burning Rate of Liquid Monopropellants for Guns," Rept. BRLTR-03018, Ballistic Research Laboratory, Aberdeen Proving Ground, April 1980.

${ }^{6}$ McBratney, W. F., "Burning Rate Data LGP 1845," Rept. BRLTR-03128, Ballistic Research Laboratory, Aberdeen Proving Ground, Aug. 1981.

'Vosen, S. R., "The Burning Rate of HAN-Based Liquid Propellants," Twenty-Second Symposium (International) on Combustion, The Combustion Institute, Pittsburgh, 1988, pp. 1817-1825.

${ }^{8} \mathrm{Zhu}$, D. L., and Law, C. K., "Aerothermochemical Studies of Energetic Liquid Materials: 1. Combustion of HAN-Based Liquid Gun Propellants under Atmospheric Pressure," Combustion and Flame, Vol. 70, Dec. 1987, pp. 333-342.

${ }^{9}$ Beyer, R. A., "Atmospheric Pressure Studies of Liquid Monopropellant Drops in Hot Flow," Rept. BRL-TR-2768, Ballistic Research Laboratory, Aberdeen Proving Ground, Oct. 1986.

${ }^{10}$ Beyer, R. A., "Single Droplet Studies in a Hot, High Pressure Environment," Rept. BRL-TR-2900, Ballistic Research Laboratory, Aberdeen Proving Ground, March 1988.

"Beyer, R. A., and Teague, M. W., "Studies of Single Liquid Propellant Drops in Hot, High Pressure Environments," 22nd JANNAF Combustion Meeting, CPIA Publication 457, Vol. 2, Oct. 1986, p. 429.

${ }^{12}$ Reitz, R. D., and Bracco, F. V., "Mechanism of Atomization of a Liquid Jet," Physics of Fluids, Vol. 25, Oct. 1982, pp. 1730-1742.

${ }^{13}$ Chatwani, A. V., and Bracco, F. V., "Computation of Dense Spray Jets," Third International Conference on Liquid Atomization and Spray Systems, Imperial College, London, 1985. 
${ }^{14}$ Ruff, G. A., Sagar, A. D., and Faeth, G. M.,"Structure and Mixing Properties of Pressure-Atomized Sprays," AIAA Journal, Vol. 27, No. 7, 1989, pp. 901-908.

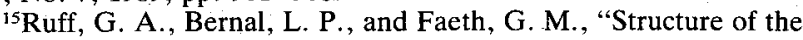
Near-Injector Region of Non-Evaporating Pressure-Atomized Sprays," Journal of Propulsion and Power, Vol. 7, No. 2, 1991, pp. 221-230.

${ }^{16}$ Ruff, G. A., and Faeth, G. M., "Continuous-Phase Properties of the Near-Injector Region of Non-Evaporating Pressure-Atomized Sprays," AIAA Paper 90-0464, 1990.

${ }^{17}$ Solomon, A. S. P., Shuen, J.-S., Zhang, Q.-F., and Faeth, G. M., "Measurements and Predictions of the Structure of Evaporating Sprays," Journal of Heat Transfer, Vol. 107, Aug. 1985, pp. 679686.

${ }^{18}$ Lee, T.-W., "Structure of Combusting Monopropellant Sprays at High Pressures," Ph.D. Thesis, Univ. of Michigan, Ann Arbor, MI, 1990.

${ }^{19} \mathrm{Gordon}, \mathrm{S}$., and McBride, B. J., "Computer Program for Cal- culation of Complex Chemical Equilibrium Compositions, Rocket Performance, Incident and Reflected Shocks and Chapman-Jouguet Detonations," NASA Rept. SP-273, Washington, DC, 1971.

${ }^{20}$ Ranz, W. F., "Principles of Inertial Impaction," Engineering Research Bulletin B-66, Pennsylvania State Univ., University Park, PA, 1956.

${ }^{21}$ Jeng, S. M., and Faeth, G. M., "Species Concentrations and Turbulence Properties in Buoyant Methane Diffusion Flames,"Journal of Heat Transfer, Vol. 106, Nov. 1984, pp. 721-727.

${ }^{22}$ Lockwood, F. C., and Naguib, A. S., "The Prediction of Fluctuations in the Properties of Free, Round Jet, Turbulent Diffusion Flames," Combustion and Flame, Vol. 24, Feb. 1975, pp. 109-124.

${ }^{23}$ Faeth, G. M., "Mixing, Transport and Combustion in Sprays," Progress in Energy Combustion Science, Vol. 13, 1987, pp .293-345.

${ }^{24}$ Spalding, D. B., GENMIX: A General Computer Program for Two-Dimensional Parabolic Phenomena, Pergamon Press, Oxford, 1978. 